Article

\title{
Sun Position Identification in Sky Images for Nowcasting Application
}

\author{
Alessandro Niccolai * (i) and Alfredo Nespoli \\ Politecnico di Milano, Dipartimento di Energia, Via La Masa, 34, 20156 Milan, Italy; alfredo.nespoli@polimi.it \\ * Correspondence: alessandro.niccolai@polimi.it
}

Received: 7 October 2020; Accepted: 13 November 2020; Published: 16 November 2020

check for updates

\begin{abstract}
Very-short-term photovoltaic power forecast, namely nowcasting, is gaining increasing attention to face grid stability issues and to optimize microgrid energy management systems in the presence of large penetration of renewable energy sources. In order to identify local phenomena as sharp ramps in photovoltaic production, whole sky images can be used effectively. The first step in the implementation of new and effective nowcasting algorithms is the identification of Sun positions. In this paper, three different techniques (solar angle-based, image processing-based, and neural network-based techniques) are proposed, described, and compared. These techniques are tested on real images obtained with a camera installed at SolarTech ${ }^{\text {Lab }}$ at Politecnico di Milano, Milan, Italy. Finally, the three techniques are compared by introducing some performance parameters aiming to evaluate of their reliability, accuracy, and computational effort. The neural network-based technique obtains the best performance: in fact, this method is able to identify accurately the Sun position and to estimate it when the Sun is covered by clouds.
\end{abstract}

Keywords: photovoltaic forecasting; nowcasting; image processing; solar position; neural networks

\section{Introduction}

The growth of Renewable Energy Sources (RES) is expected to increase in the following years; among all the sources, the expansion is going to be led by solar photovoltaic (PV) at both the distributed and centralized levels [1]. The penetration of non-programmable RES is posing great technical challenges to grid operators in terms of reliability and stability of the overall system [2]. PV resource, in fact, is strongly dependent on meteorological conditions such as stochastic movements of clouds, fog, and other meteorological parameters that hinder solar radiation $[3,4]$.

On a national level, a portfolio of different power plants with varying degrees of responsiveness is required to meet the continuously changing demands. However, while the aggregate load fluctuations vary within few percentage around the expected values, the power generation from plants exploiting RES presents larger and generally faster variations. For this reason, the shortness they can cause is usually faced with some "hot standby" spare capacity, which affects the overall efficiency of the system [5]. For what concerns microgrids, the RES fluctuation is usually compensated by energy storage and backup technologies, which should be properly sized and coordinated, generally to increase efficiency, to reduce fuel consumption, or to wisely combine the two [6,7].

In this scenario, an accurate PV power forecast can greatly help operations; unit commitment; and, on a longer time horizon, sizing of available units, ultimately reducing investment costs [8]. Selecting the more suitable prediction model in terms of accuracy is a big issue related to the forecasting topic. The available 
methodologies and their performances are highly affected by the selected time horizon and the task to be accomplished $[9,10]$. In fact, when PV modules are part of the generation units of a complex energy system, an integrated forecasting approach is useful to address several scopes. From the Energy Management System (EMS) point of view, a 24-h-ahead forecast is needed to initially perform strategic optimization of system management, with the goal of, for example, minimizing the operating cost, reducing the fuel consumption over the following $24 \mathrm{~h}$, and properly managing and sizing the battery energy storage system (BESS) [11,12]. Approaching the operating time, additional refinements are useful to further correct the original forecast and to tune the dispatching schedule in the so-called intraday forecast [13]. Eventually, during the operations, integration of the previous forecast for the following minutes is required for predictive power management [14,15].

The importance of short-term forecasting (in the range from 0 to $30 \mathrm{~min}$ ), called nowcasting, is growing due to regulatory issues [16] and for optimal sizing and management of storage [17]. In nowcasting, the main issue is related to identification of the sudden spikes and drops in PV power production. These fluctuations are mainly related to the presence of moving clouds, which hinder solar radiation. For this reason, most of the work found in the literature relies on the adoption of satellite images and whole sky images [18-21]. Other works rely on machine learning algorithms to serve the scope [22].

All the abovementioned approaches are usually compared with a benchmark, which is represented by the persistence. A persistence forecasting method consists of imposing the next value of the forecast parameter to be equal to the last measured one [23]. In the very short term, this method achieves very good results, especially in stationary conditions, and presents the advantage of a negligible computational cost. Nevertheless, the validity of persistence loses effectiveness as the forecast horizon increases [24].

The aim of this paper is to introduce, describe, and compare three different Sun identification techniques that can be applied to the nowcasting problem. This paper extends the preliminary analysis provided in [25], providing more details on the motivations, deepening the investigation of the implemented techniques, and introducing some new numerical performance parameters that are used to test the implemented techniques against manual recognition.

The artificial neural network-based method-proposed technique is able to overcome the problem of finding the true optical distortion of the image acquisition system, often unknown in industrial applications. In fact, this method exploits the solar angle information that avoids, after the training process, complex and time-consuming image-processing operations.

This paper is structured as follows. In Section 2, an analysis of sudden changes of the irradiance value that motivates the use of sky images for nowcasting is provided. Then, in Section 3, the three different identification techniques are described in detail. Section 4 shows a numerical and a qualitative comparison between the three approaches, and finally, in Section 5 some conclusions are drawn.

\section{Motivations}

As mentioned earlier, nowcasting is very important for better management of the grid with a higher PV penetration.

\subsection{Sudden Changes in Solar Irradiance}

Among other techniques, the analysis of sky images is very promising for nowcasting applications, since it is able to detect local phenomena that can create sudden ramps in the irradiance level and, thus, in PV power production.

In particular, this technique is very important for forecast refinement in those conditions when sudden weather changes occur. In Figure 1, two exemplifying graphs are provided for typical sunny (Figure 1a) and cloudy (Figure 1b) days. Here, the measured GHI (Global Horizontal Irradiance) and the clear-sky 
radiation are represented in blue and light blue, respectively. In these conditions, since the variation among successive minutes is not large, a persistence model is able to accurately grasp the irradiance behavior.

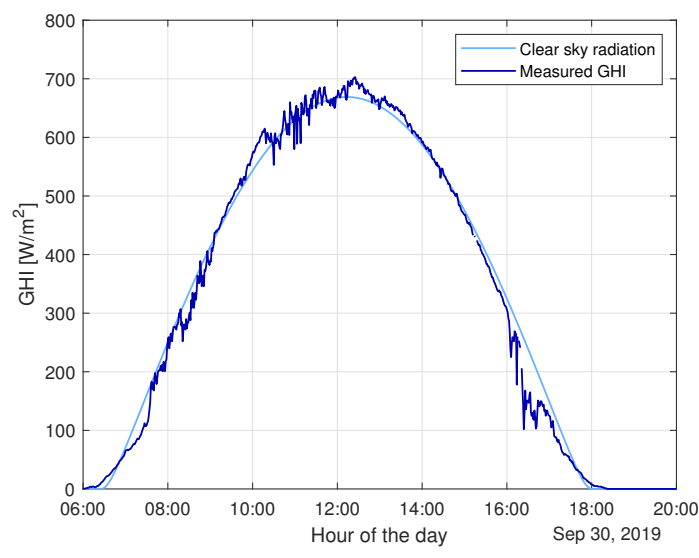

(a)

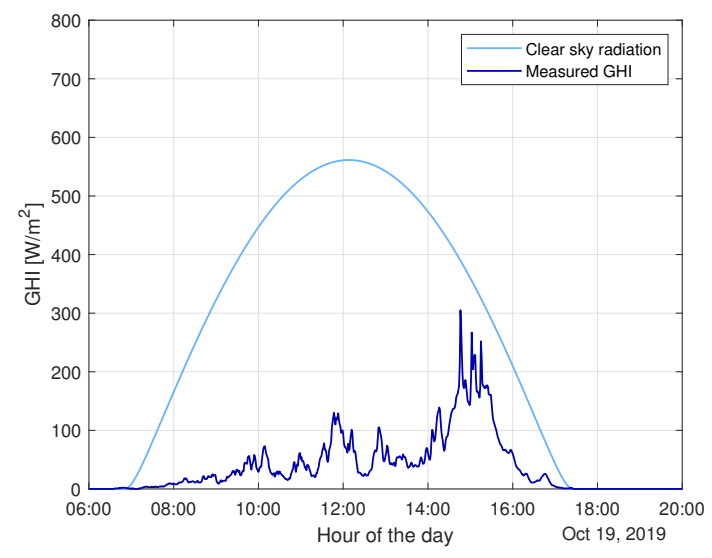

(b)

Figure 1. Measured GHI (Global Horizontal Irradiance) and computed clear-sky radiation for sunny (a) and cloudy (b) days measured at SolarTech ${ }^{\text {Lab }}$ of Politecnico di Milano.

On the other hand, Figure 2 shows a very different condition in the morning hours. In this period, in fact, high variation in the irradiation measurements is observable with sharp spikes and drops. As can be seen, in the range of a few minutes, the irradiation goes from over $700 \mathrm{~W} / \mathrm{m}^{2}$ to $150 \mathrm{~W} / \mathrm{m}^{2}$. These sudden ramps depend on small moving clouds and their relative position with respect to the Sun and the measuring device and are very hard to be identified with statistical and regressive techniques.

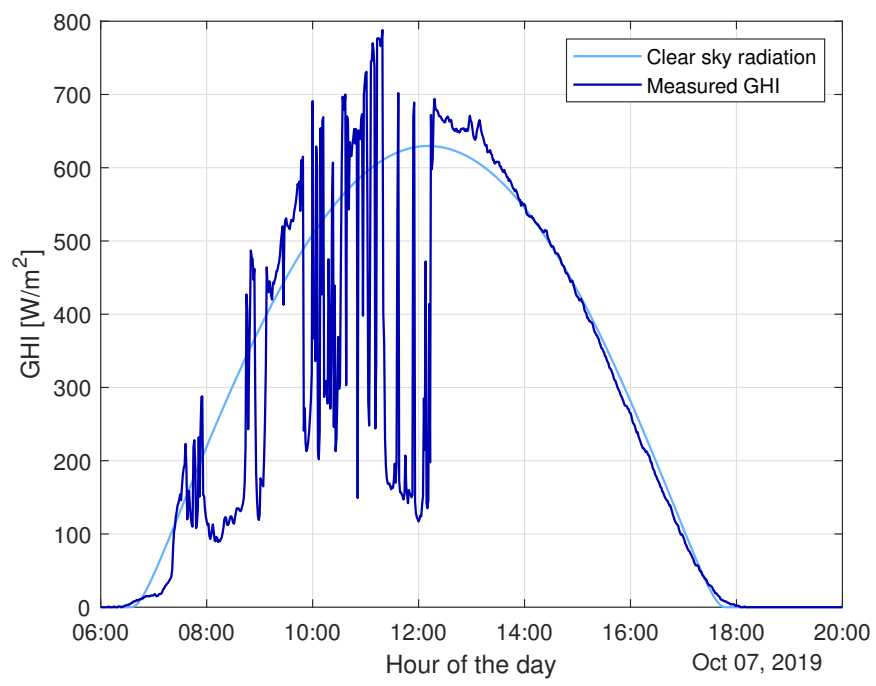

Figure 2. Measured GHI and clear-sky radiation on a variable weather day.

Following this, a day-ahead forecasting is even less capable of identifying these local phenomena. For example, Figure 3 compares the measured GHI and the irradiation forecast performed through a persistence model for the following 15 minutes. The cumulative plot of the committed absolute error is shown in Figure $3 \mathrm{~b}$. Despite $80 \%$ of the available samples providing an error lower than $100 \mathrm{~W} / \mathrm{m}^{2}$, mainly due to the regular trend visible for the afternoon, a nonnegligible percentage of the samples (10\%) return an error above $300 \mathrm{~W} / \mathrm{m}^{2}$ and $3 \%$ of the samples return an error above $500 \mathrm{~W} / \mathrm{m}^{2}$. 


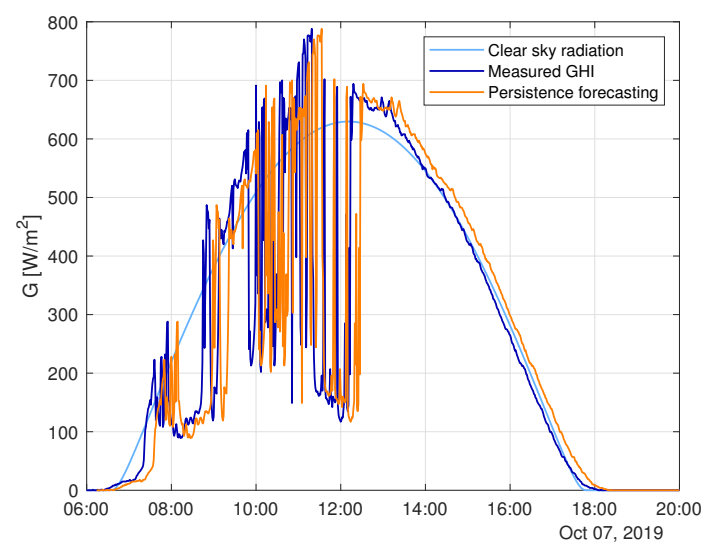

(a)

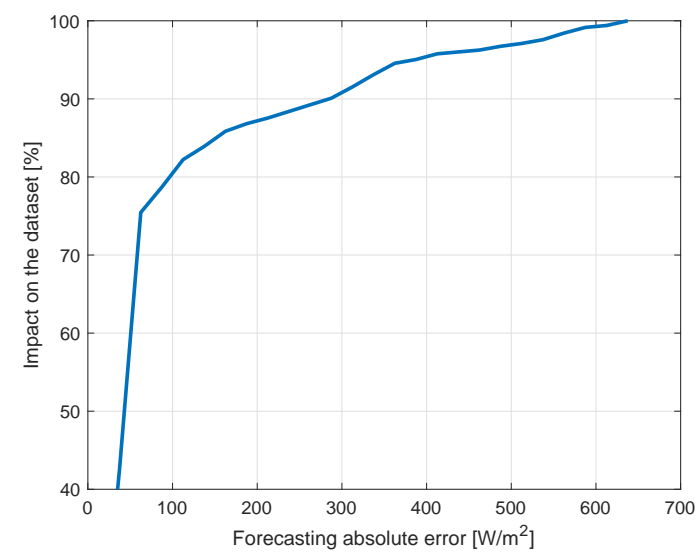

(b)

Figure 3. Persistence forecasting with 15 minutes of time horizon: irradiance values (a) and cumulative error plot (b).

These high error values highly affect the grid and microgrid operation and pose severe implications from the power management point of view in spite of their marginal contribution in terms of energy.

\subsection{Nowcasting by Means of Sky Images}

In this scenario, infrared sky images can effectively be implemented for this application because they can provide information on local phenomena with a very high accuracy. The use of these images is able to overcome some problems of true color. The most important one is related to the color saturation: infrared images are less impacted by the presence of the Sun, as shown in Figure 4. Here, a comparison between the two types of images at the same time instant is shown.

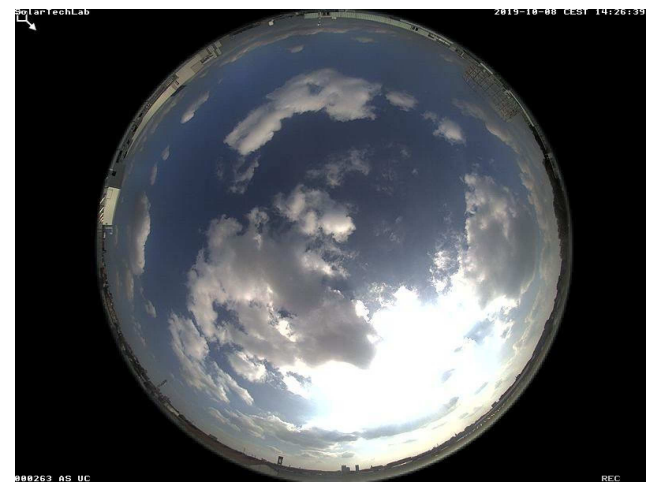

(a)

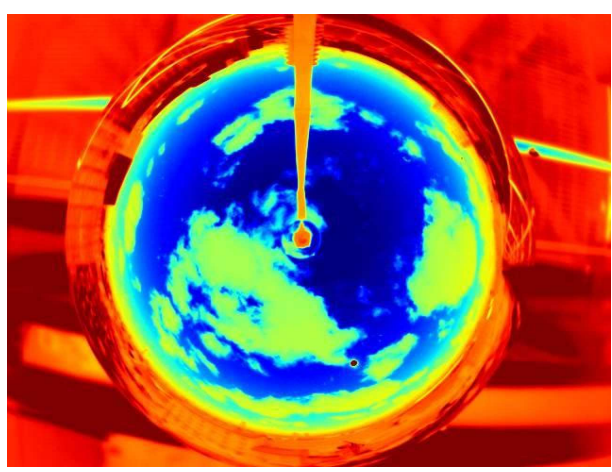

(b)

Figure 4. Comparison between sky images: visible (a) and infrared (b) spectrum.

The infrared image (Figure $4 \mathrm{~b}$ ) uses false colors to increase the contrast between the clear-sky (in blue) and the cloud coverage that can range from green to red according to the different types of clouds. In these images, the Sun is shown as a well-defined black dot. 
The system for image acquisition is installed at SolarTech ${ }^{\mathrm{Lab}}$ [26] in Politecnico di Milano, and its concept is shown in Figure 5. It is composed by an infrared thermal camera pointing toward a hemispherical mirror that reflects the sky providing an all-sky image.

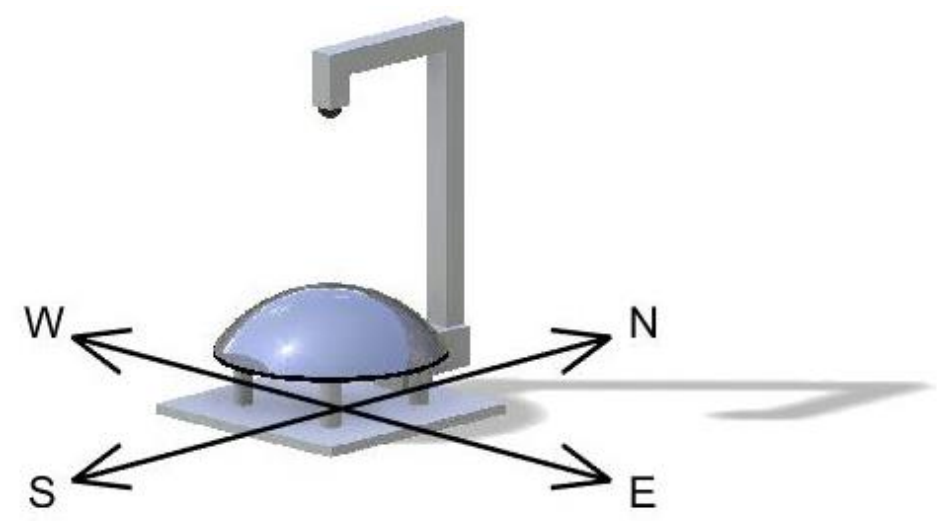

Figure 5. Acquisition system for the sky images: the camera (in black) records the sky image that is reflected on the convex mirror. The orientation of the device is also indicated.

The camera arm is orientated and placed to the north in order to have the half-circumference in which the projection of the Sun is expected free from obstacles and unwanted shadows. The zenith is placed in the central position of the image.

Next to the camera, a weather station is installed, allowing to acquire different ambient measurements. The full details of the available instrumentation can be found in [27]. In the present work, measurements of GHI are used. This information, synchronized with the available images, compose the complete database. Both the available cameras provide a frame per minute and allow for analysis of the cloud movement and, thus, forecast of sudden ramps. The GHI is acquired at higher frequency (10 s), and only the samples synchronized to the camera sampling are used.

\subsection{Solar Position Identification}

In order to adopt sky images for nowcasting application, identification of the Sun position is a key aspect: in fact, the presence of clouds is the factor that hinders most of the power production from the solar source. In particular, those clouds that interpose themselves between the solar disk and the PV system are the ones that affect production. In Figure 6, a variable weather day is given and two different time instants are singularly analyzed. In the first one (orange rectangle, relative to 10 A.M.), the cloud coverage is very high and is greatly above $50 \%$ with respect to the overall sky vault. Despite this, the Sun is not directly obstacled and the measurements of irradiance are above clear sky due to multiple reflections; on the other hand, in the second one (green rectangle), in spite of an apparent near-to-clear-sky condition, a low value of irradiance is measured. 


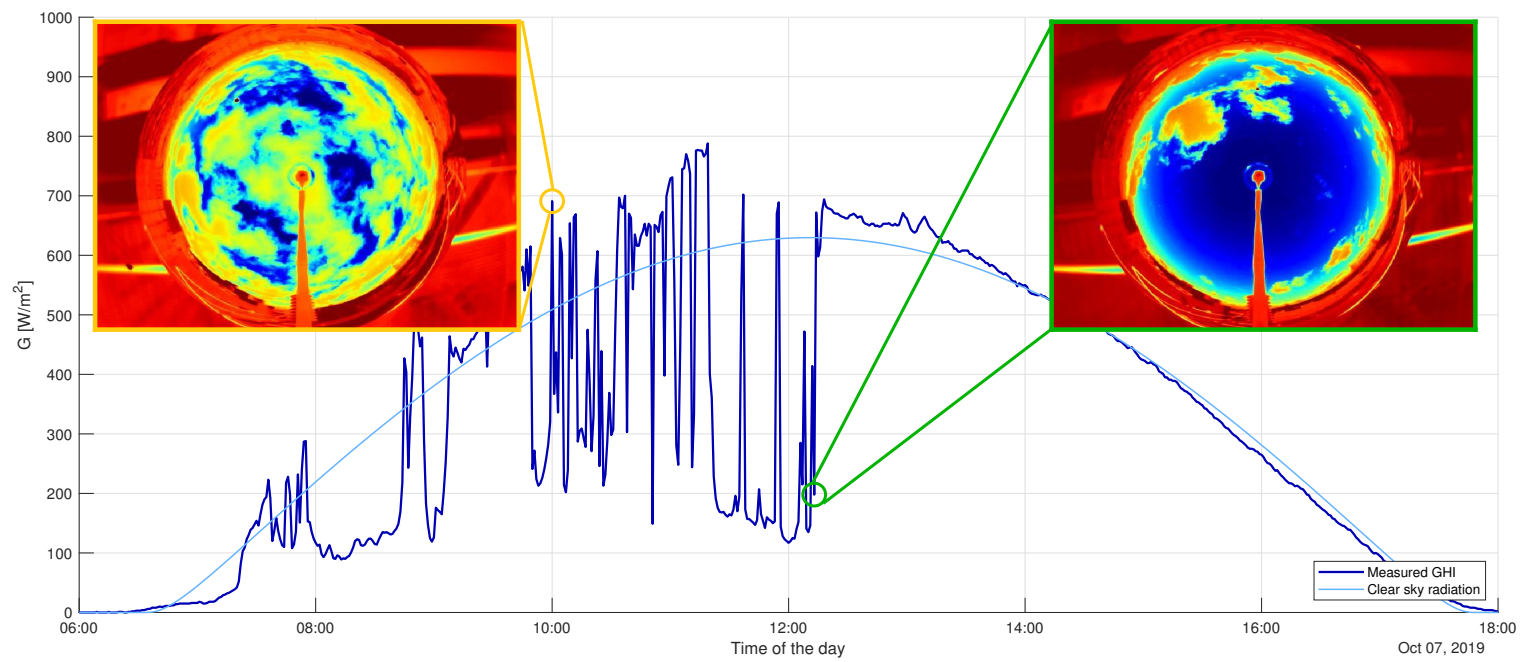

Figure 6. Cloud effect on irradiation measurements.

It is then clear that the irradiation measurement is directly correlated to the presence of clouds that directly cover the Sun. In Figure 7, the two previously analyzed frames are shown together with a zoom of the surroundings of the Sun. As it is seen in Figure 7b, the Sun appears smaller with respect to Figure 7a because it is partially shadowed by clouds.

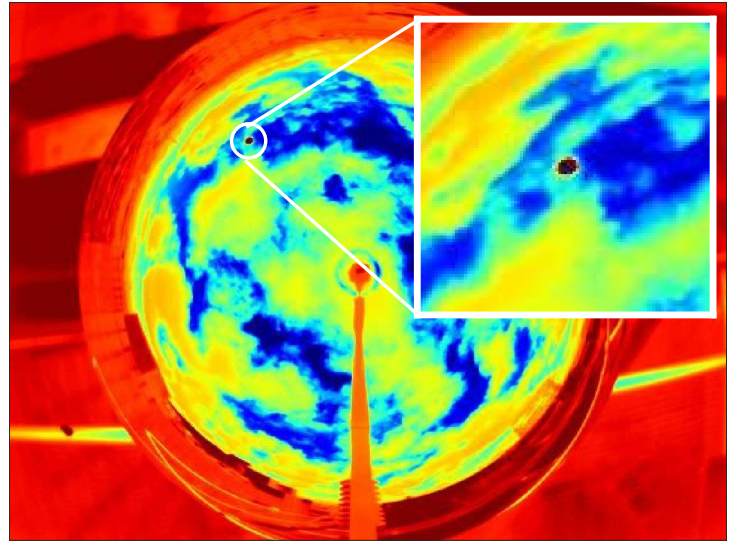

(a)

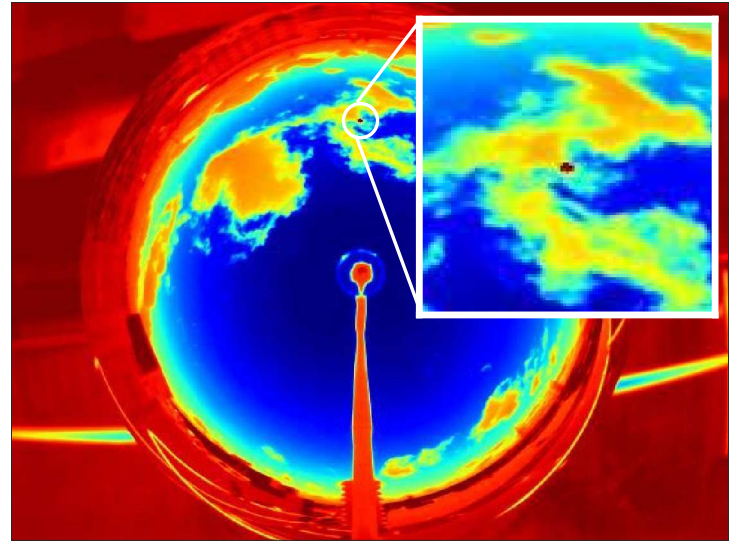

(b)

Figure 7. Detailed images of the surroundings of the uncovered (a) and partially covered (b) Sun.

In view of the foregoing, the first step to be performed when analyzing the images for nowcasting applications is identification of the solar position. In particular, both accuracy and reliability in the position identification of the Sun are key aspects. In fact, since the Sun is composed of a few pixels on the image, small inaccuracies in the Sun identification can cause wrong ramp detection. On the other hand, the adopted methodology must be able to detect the solar position in every weather condition, even when clouds could hinder the view. 


\section{Methods}

As said in the previous sections, PV nowcasting based on sky images is fundamental in spotting the solar behavior, such as spikes and drops in the measured irradiation that could hardly be identified through a purely statistic methodology.

In this paper, three different methods have been implemented, with increasing computational complexity and accuracy.

The first method exploiting information regarding the theoretical solar position provides the projection of the Sun on the image through a mathematical representation.

The second implemented method identifies the solar position by means of image processing. The overall accuracy of the method is higher than the previous one, though requiring more computational effort. The main drawback of this approach is the impossibility to identify the Sun when it is covered by clouds. Finally, the third method is based on Artificial Neural Networks (ANNs) that are able to infer the correlation between the theoretical solar angles and the solar position on the images; the strength of this approach is that, regardless of the weather conditions, it is able to provide the correct position of the Sun.

In the following sections, these methodologies are described in details.

\subsection{Solar Angle-Based Identification}

The first method relies on the solar angles that are commonly used to determine the Solar position in the sky.

The solar angles (azimuth $\gamma$ and elevation $\alpha$ ) can be determined as function of the coordinates of the location and of the time of day. Their determination has been performed as presented in [28] and implemented in [29].

Once $\alpha$ and $\gamma$ have been computed, the approximated solar position on the image can be found applying the following trigonometrical relations:

$$
\mathbf{x}_{S}=\mathbf{x}_{0}+\left[\begin{array}{c}
-L \cdot \sin (\gamma) \cdot \cos (\alpha) \\
L \cdot \cos (\gamma) \cdot \cos (\alpha)
\end{array}\right]
$$

where $L$ is the radius on the image and $\mathbf{x}_{0} \in \mathbb{R}^{2}$ is the two-dimensional vector for the central position on the image:

$$
\mathbf{x}_{0}=\left[\begin{array}{l}
x_{0} \\
y_{0}
\end{array}\right]
$$

Figure 8 shows an example of the application of this technique. The black line represents the theoretical Sun trajectory in the sky, the upper yellow dot is the real position of the Sun, and the lower dot is its projection on the image; the central position is the point where the two red cross. The solar angles can be identified as follows: the orange arc represents the azimuth $\gamma\left(0^{\circ}\right.$ north, increasing towards east), and the purple one is the elevation $\alpha$ ( $0^{\circ}$ representing the horizon, positive upwards).

This technique allows one to find the approximate solar position with simple trigonometric operations. Moreover, once the central position of the images is given, no further information regarding the images are needed. On the other hand, due to the optics and mirror surface distortion, unknown in the current work as well as in many industrial situations, it can only provide a rough estimation of the actual solar position.

The main advantage of this technique is hence the ease of determination of the solar position since it can be done without complex analyses or a historical dataset. 


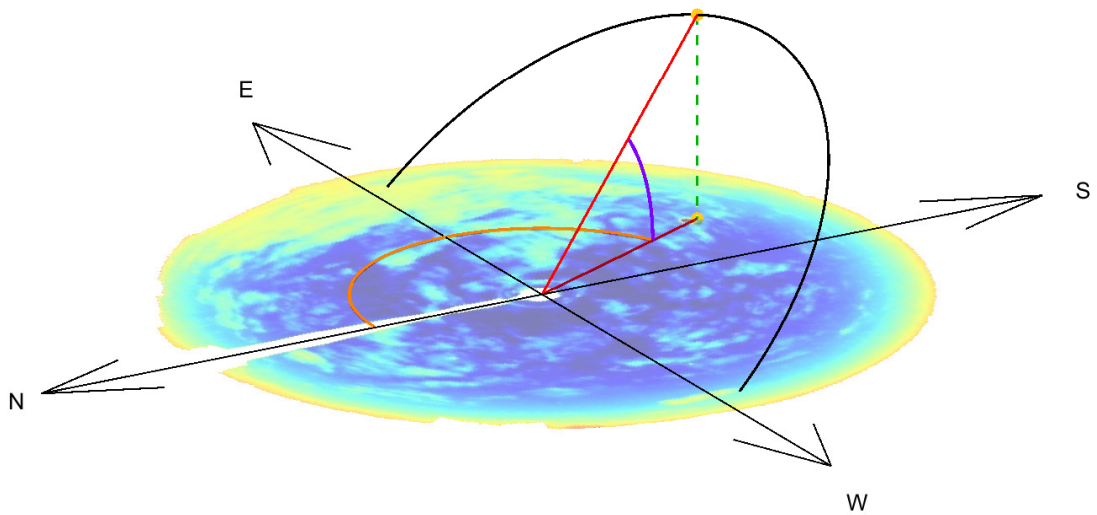

Figure 8. Representation of solar angles used: azimuth is shown by the orange line, while the elevation is shown with the purple one.

\subsection{Image Processing-Based Identification}

The second methodology is based on image processing algorithms, and it is capable of identifying the Sun by means of its shape and color.

The first step to be applied on the available images consists of a color threshold filtering, where the thresholds are imposed on all the pixels and color channels and their values are set for the specific case under study in order to identify the Sun. On the current images, in fact, the Sun appears as a dark dot. Figure 9a shows the color filtering result overlapped on a sample image: the whiter areas are the identified one as a candidate for the solar position, while the darker areas are discarded. As it is seen, there are large areas that belongs to the background and are not interesting to the scope. For this reason, thanks to the rigid geometry of the camera, it is possible to eliminate these areas applying a mask. Figure $9 \mathrm{~b}$ shows this mask: the central part (lighter) is the correct sky image.

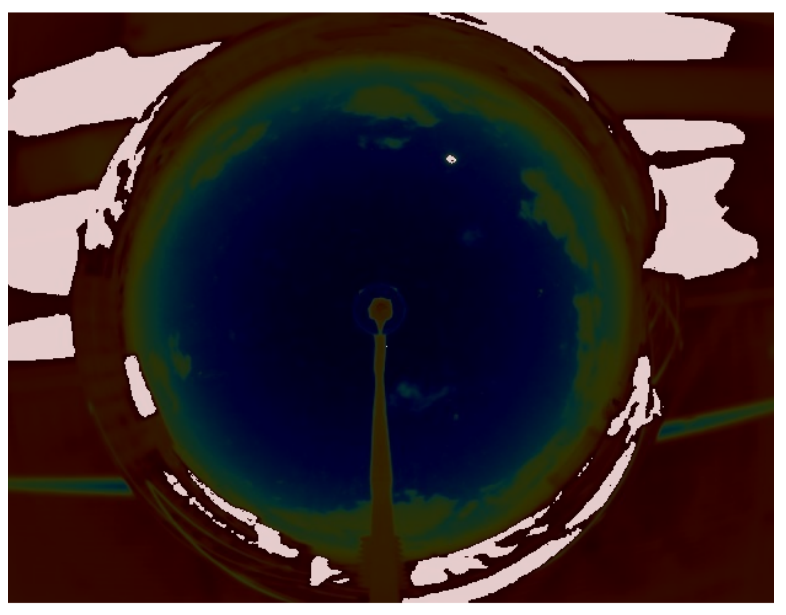

(a)

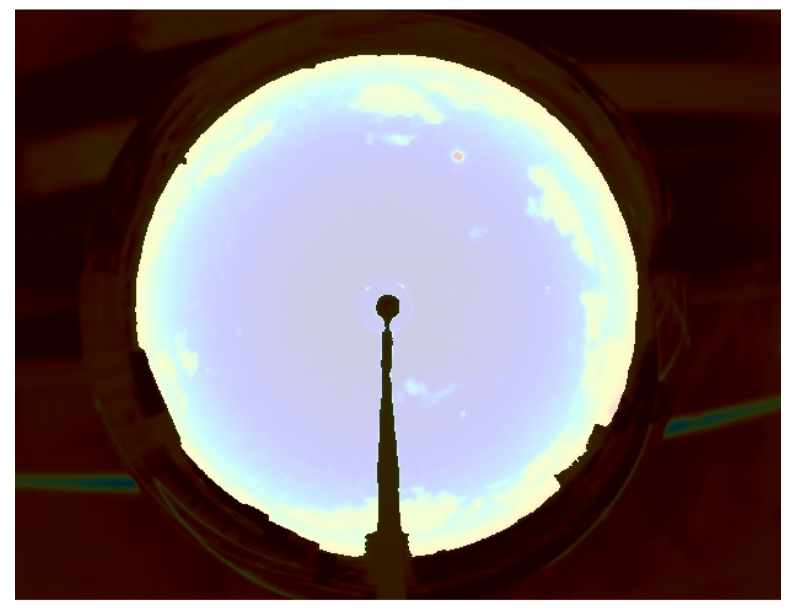

(b)

Figure 9. First two steps of image processing: (a) color thresholding filtering process and (b) background elimination.

Finally, the two combined filters (the first one constructed ad hoc for the frame under study, Figure 9a, and the second one common for all images, Figure $9 b$ ) are combined, returning some candidates eligible 
for the solution of the problem. Unfortunately, depending on the boundary effect due to the presence of the supporting pole for the camera and on the sky color variations, more than one identification can often be returned. To select the true identification, a position control is applied: information about the solar angles is exploited, and the search of possible locations of the Sun is reduced to an elliptical area surrounding of the position detected through the methodology explained in Section 3.1. The choice of the elliptical shape is consequent to the fact that the error committed on radial distance from the central position $\mathbf{x}_{0}$ is greater than the error on the azimuth angle.

Figure 10 shows an example in which candidates were found. If the reduction of the search space were not applied, it would have not been possible to identify the correct solar position. On the other hand, the ellipse allows for proper identification of the desired solution, the only one in the elliptical shape.

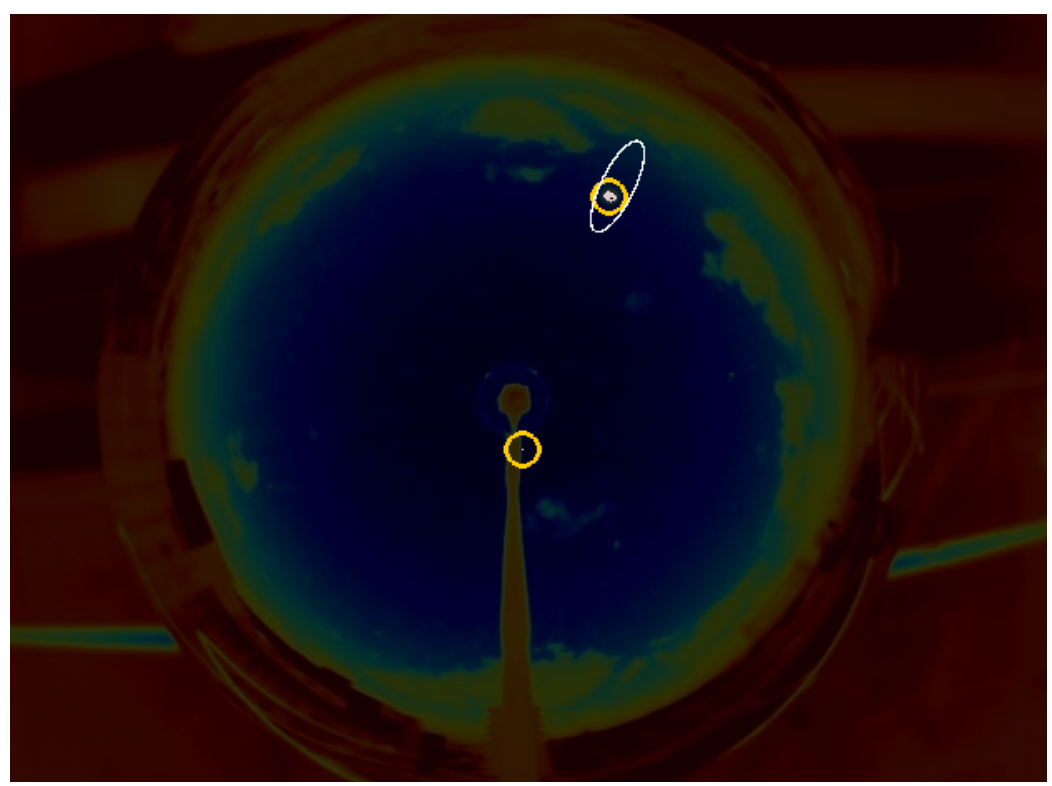

Figure 10. Reduction of the space for the Sun location.

In some cases, such as the ones depicted in Figure 11, two situations in which the algorithm returned multiple outcomes are presented. In Figure 11a, two candidates are found, both of them false positives being outside the search space. In this case, the actual position of the Sun cannot be detected by the algorithm because it is hidden behind the clouds.

The opposite situation shown in Figure 11b, typical of rainy days, represents multiple candidates found in the ellipse. Due to the impossibility of detecting the real position and in order to adopt a conservative approach, no Sun position is detected here.

Even if it can lead to some errors, this method is generally very accurate and the computational time required to process the images is quite limited.

The main drawback of this technique is related to the impossibility of finding the Sun when it is covered by clouds: this technique, in fact, is conservative and provides the Sun position only when the estimation satisfies the accuracy conditions modelled by the set of filters shown before. This is an important drawback because cloudy days are very important conditions that cannot be excluded from the study. 


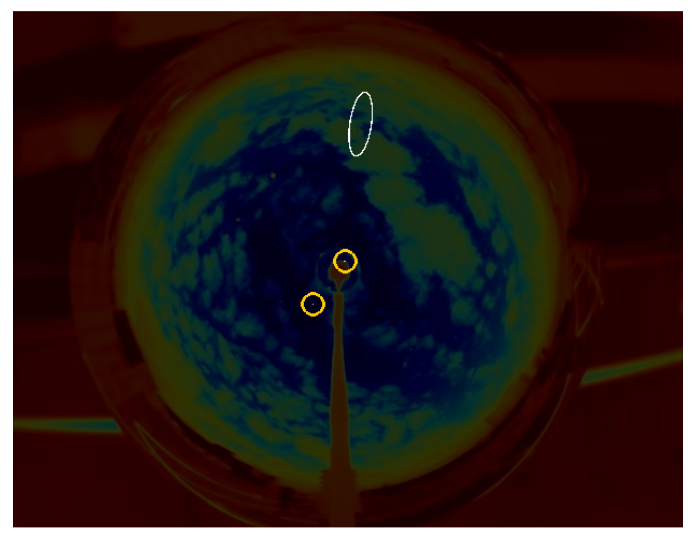

(a)

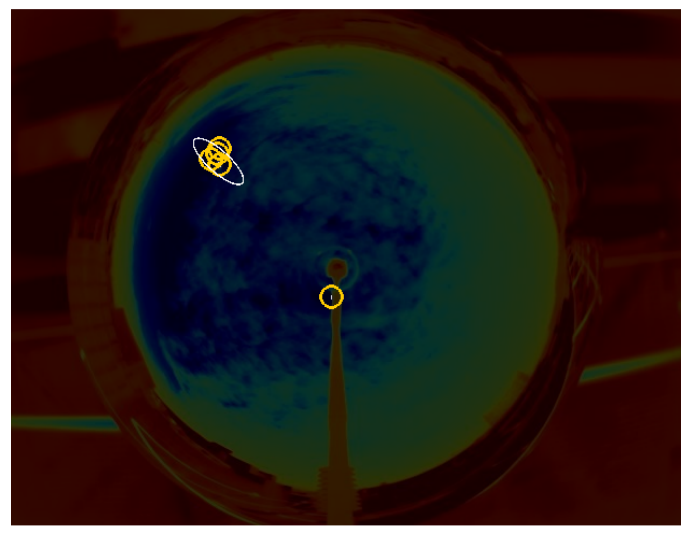

(b)

Figure 11. Other possible cases for Sun identification: (a) no spot in the ellipse and (b) several spots in the ellipse.

\subsection{ANN-Based Identification}

The third methodology aims at overcoming all drawbacks of the previously analysed techniques, i.e., the solar angle-based and the image processing-based. In particular, this methodology exploits the capability of Artificial Neural Networks (ANNs) learning from historical data without information concerning the system.

The ANN receives the solar angles $(\alpha$ and $\gamma$ ) as input and returns the $x$ - and $y$-coordinates of the Sun on the image as output. In this way, the neural network is able to eliminate the deformation introduced by the mirror and the optics of the camera.

Figure 12 shows the scheme of the neural network working process: the inputs of the network are solar angles (azimuth and the elevation), and the outputs are the $\mathrm{x}$ - and $\mathrm{y}$-coordinates of the Sun on the image.

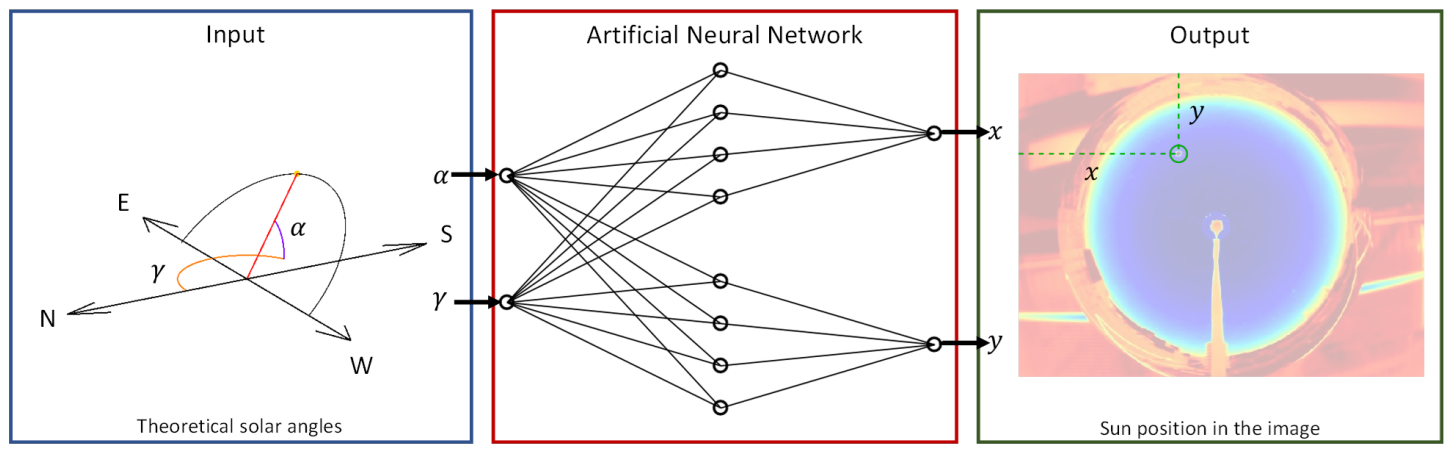

Figure 12. Working principle scheme of the neural network-based identification method: the network receives as inputs the azimuth and the elevation and returns as outputs the the $x$-and $y$-coordinates of the Sun on the image.

The aforementioned choice of inputs is not the only possible. Day and time could be used as well, but their adoption requires a higher number of historical data to let the network generalize the overall trend.

The implemented neural network has 10 hidden neurons for identification of both the $x$ - and the $y$-coordinates. The training and test sets were obtained using an image dataset of 30 days. The outputs corresponding to the inputs are obtained through the image processing methodology explained above. 
Consequently, those samples where the image-processing fails at identifying the solar position were rejected and excluded from the training dataset. Finally, the overall number of available samples was equal to 9774.

The training process of the neural network was performed with $70 \%$ of the dataset, while $10 \%$ was used for validation. The remaining $20 \%$ was used for testing the system performance.

Due to the intrinsic stochasticity of the training process and in order to overcome possible local minima in the optimization process, 10 independent trials have been performed with a different subdivision of the training, validation, and test sets: the accuracy in all of them was very high, proving the robustness of the approach.

The main limitation of this methodology is related to the required training set: in fact, it is important to cover the entire range of possible solar angles because ANNs are very effective in interpolation but less accurate in extrapolation. However, the choice of the solar angles as the input reduces the number of required training days.

\section{Results and Discussion}

In the present section, the three above-described techniques are compared using the available dataset. In addition, a manual identification of the Sun position was conducted over the 30 available days to be used as a benchmark in the comparison.

In order to compare the performances of the solar identification techniques, three performance parameters were introduced. The first one, counting the number of revealed samples, provides an indication in terms of availability of the approach. In fact, it is desirable that the Sun position is identified in as many cases as possible. The second performance parameter, counting the wrong identifications with respect to manual analysis, provides information regarding the reliability. Two aspects were considered in this regard. Firstly, an identification is wrong if it is more than 3 pixels away from the manual one. Secondly, for the image processing-based technique, the number of false identifications is counted too (the Sun was not visible to the human eye, but it was detected by the algorithm). The choice regarding the number of pixels derives from the consideration that, through manual identification, the central position of the Sun was identified, and the solar disk can be greatly approximated by a circumference with radius equal to 3 pixels. Hence, all those outcomes inside this shape are considered correct.

Finally, the last performance parameter takes into account the time needed to identify the solar position for a whole day; for the neural network analysis, the training time ( $4.85 \mathrm{~s})$ has not been taken into account because it is considered an initial overhead time, since it can be previously performed offline.

In Table 1, the numerical comparison between the three techniques is given.

Table 1. Performance parameters for the three types of automatic identifications and the manual analysis.

\begin{tabular}{cccc}
\hline Technique & Number of Samples & Wrong Identification (\%) & Time (s) \\
\hline Solar angle-based & 20,394 & 82.9 & 0.1 \\
Image processing-based & 9773 & $3.30(0.03+3.27)$ & 7.7 \\
Neural network-based & 20,394 & 0.01 & 0.3 \\
Manual analysis & 10,561 & - & 720 \\
\hline
\end{tabular}

The number of identifications for the solar angle-based and neural-network recognitions corresponds to the total amount of samples in which the solar elevation is greater than zero. As for manual identification, this represents the number of frames in which the user was able to clearly identify the solar position. Finally, the number of samples identified by the image-processing technique is related to the presence of cloud coverage or high color distortion in early morning or late afternoon. In fact, the entire image 
processing procedure and the related user-defined parameter are set as a trade-off to reduce the number of false identifications while, on the other hand, not penalize the number of detected samples. It was finally decided to set them in a conservative way, with the aim of containing the false positive, with the identification being further used in the ANN training process. With respect to manual recognition, the number of detected samples is $7.46 \%$ less.

As for identification accuracy, worth highlighting is the poor performance of the solar angle-based method. This can be explained considering that the actual mirror geometry is not known. For this reason, the projection of the Sun on the images is not well-described by the polar coordinates. More effort could be put in the mathematical description that could finally lead to greater accuracy, that though could hardly reach the image processing accuracy. This methodology, in fact, allows for identification of the Sun with an accuracy of $99.07 \%$. Despite this, the image process suffers from wrong identification, that is, the false identification in those images where the Sun is not visible. This mainly happens during rainy days, when drops of water on the reflectance surface hinder recognition. In general, these phenomena do not highly impact the overall effectiveness of the method but can generate disturbance and noise in the dataset used for the ANN learning process and must not be ignored. The ANN-based methodology is finally the method that returns the higher accuracy.

For what the required time is concerned, image processing is slightly slower than all the other automatic identifications ( $7.7 \mathrm{~s}$ with respect to the other techniques that require less than a second), but all of them are much faster than manual recognition. Worth highlighting is that solar angles and ANN -based methodologies are not dependent on the real-time acquired images but rely on theoretical information computed a priori and are ultimately dependent on the location and time.

In Figure 13, the distance in terms of pixels from manual identification is provided for the 3 methods. As it possible to see, the solar angle methodology provides a near constant distribution in the range 0 to 9 pixels. On the other hand, both the image processing and the neural network-based techniques are able to provide good approximation of the solar position, with most of the identifications in the range 0 to 1 pixel.

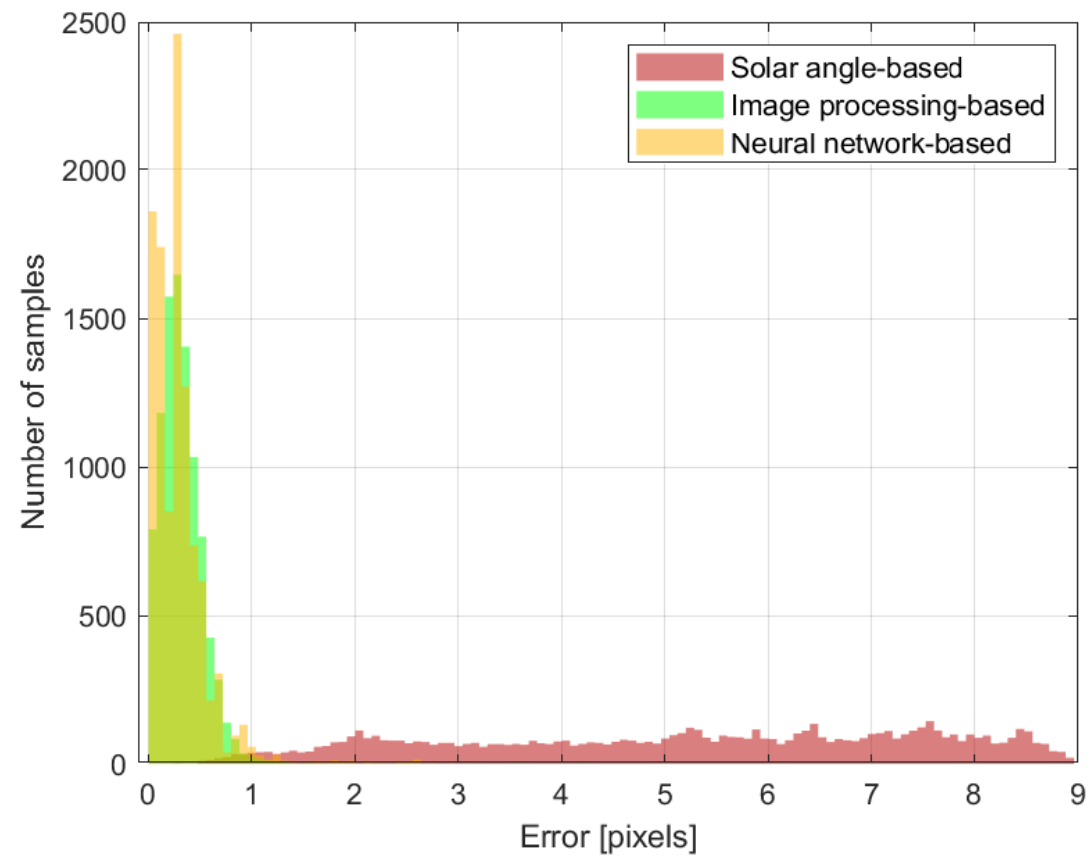

Figure 13. Distribution of errors (in pixel) of the three techniques. 
In Table 2, the information is numerically expressed in terms of mean and standard deviation ( $\mu$ and $\sigma$, respectively) of the error committed by the three presented methodologies. Worth highlighting are the results obtained for both the image processing and neural network-based techniques. The neural network, in fact, is able to provide the same degree of approximation and accuracy through proper generalization of the solar path on the images.

Table 2. Uncertainty analysis.

\begin{tabular}{ccc}
\hline Technique & Mean $(\mu)$ & Standard Deviation $(\sigma)$ \\
\hline Solar angle-based & 7.87 & 5.80 \\
Image processing-based & 0.32 & 0.22 \\
Neural network-based & 0.30 & 0.28 \\
\hline
\end{tabular}

To further understand the above results, some images are presented and their results are explained. Figure 14 shows the solar paths identified by the three methods in near-clear-sky conditions: the red line is the solar angle-based identification, the orange line is the image processing-based, and the green line is the neural network-based methodology. The ANN trajectory is taken as reference thanks to the accuracy shown with respect to manual identification. As it is possible to see, the solar-angle-based technique shows the lowest performance, especially at the beginning and at the end of the day, while the two other trajectories mostly overlap.

The image processing-based method has some holes in the early afternoon due to the presence of clouds, and it is less capable of finding the Sun position at sunrise and sunset. In fact, in these conditions, the solar position can hardly be identified due to deformation of the shape and of the colors induced by the mirror.

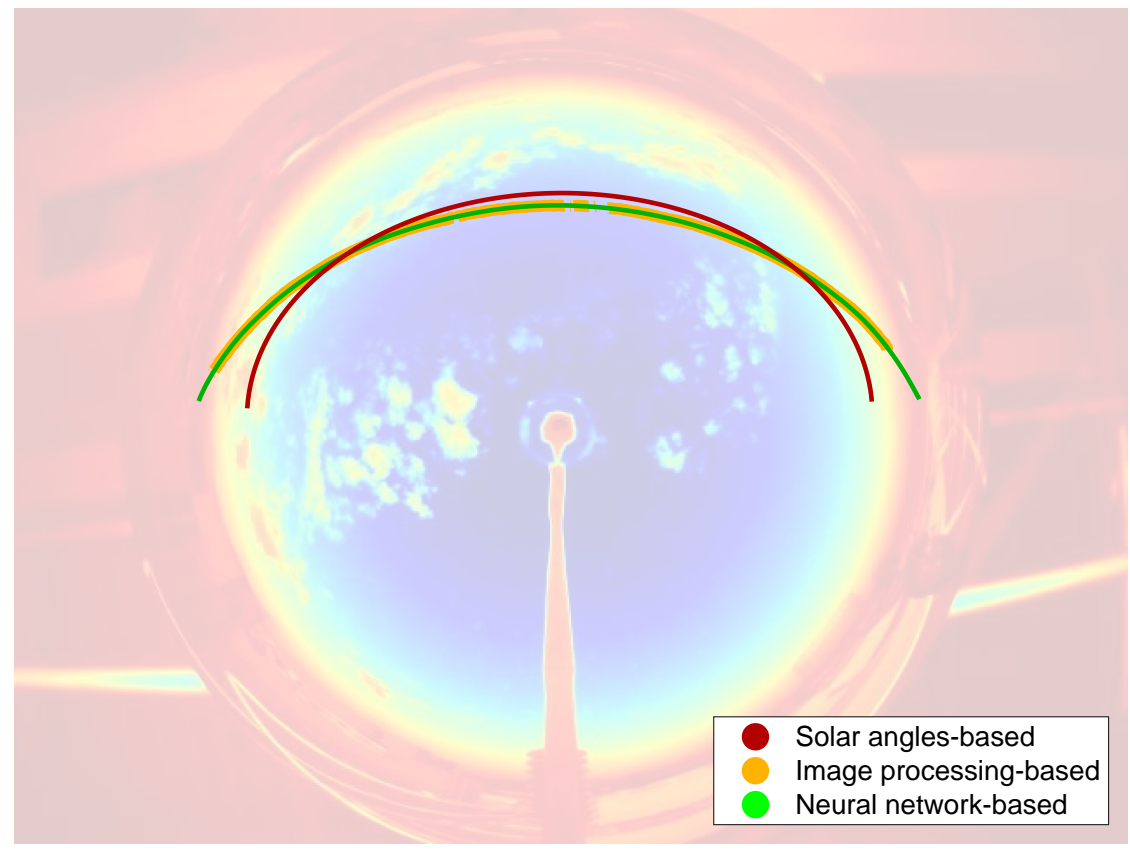

Figure 14. Trajectories identified by the three methods.

Figure 15 shows four different time instants of the same day. The time is synchronized with the Italian winter time $(\mathrm{GMT}+1)$. For each image, the area around the position of the Sun has been magnified in the top right corner of each picture. In these images, the overall poor performance shown in Table 1 of 
the solar angle-based method is confirmed: in fact, as it is possible to see, the red circles hardly overlap the other two in most of the images throughout the day. Figure $15 \mathrm{~b}$ shows that identification of the image processing-based method and of the neural network-based are almost coincident; this allows us to conclude that the learning process has been conducted properly and that the neural network learned the ability to infer the specific desired trend.

In Figure 15a,d the solar position has been correctly identified by the neural network, while the image processing-based identification failed. In these images, in fact, the Sun is clearly evident in the zooms, but due to color distortion, image processing failed at identifying the position.

Finally, Figure 15c shows a sample in which clouds cover the Sun. The neural network-based method can identify a candidate position analyzing the trajectories. The result is proven to be very close to the expected real Sun position.

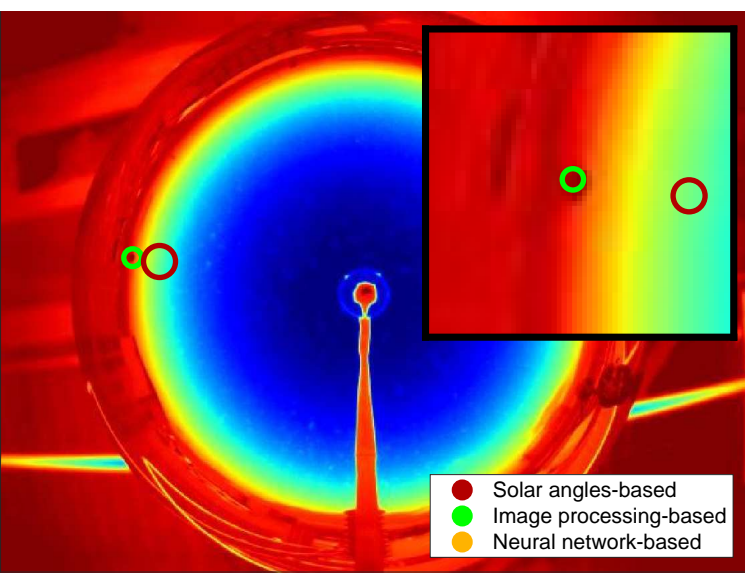

(a) Time: 06:55

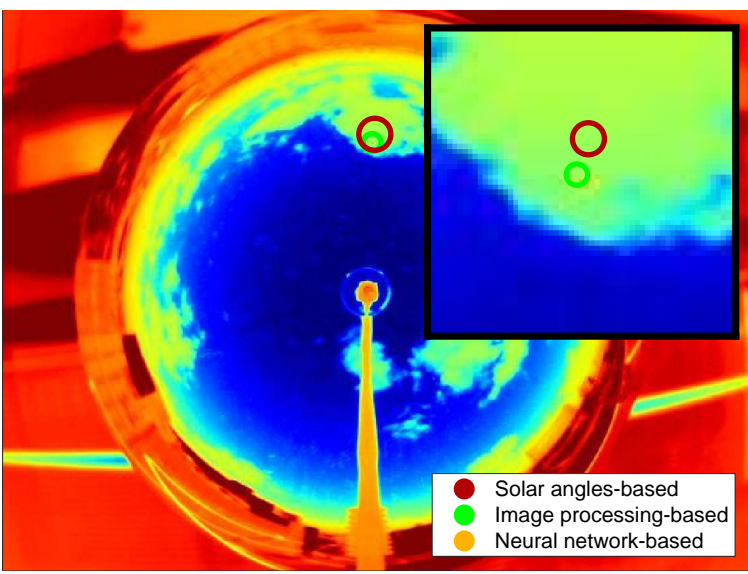

(c) Time: 12:22

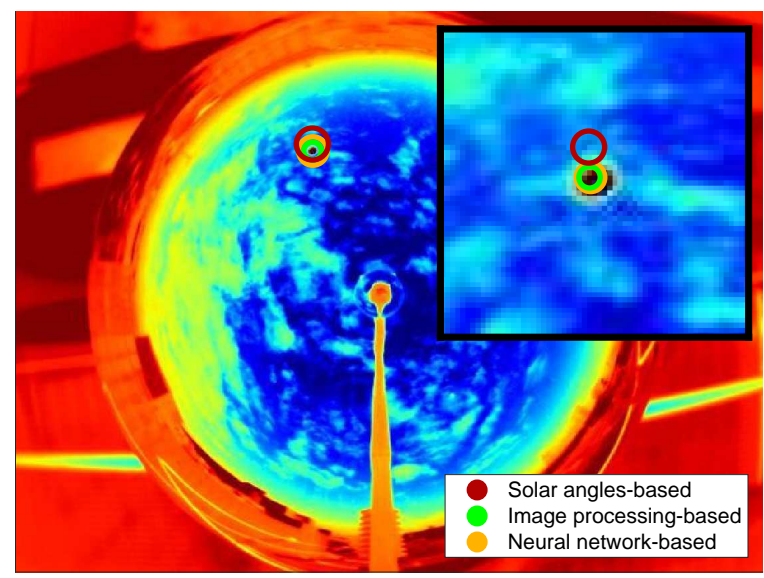

(b) Time: 10:59

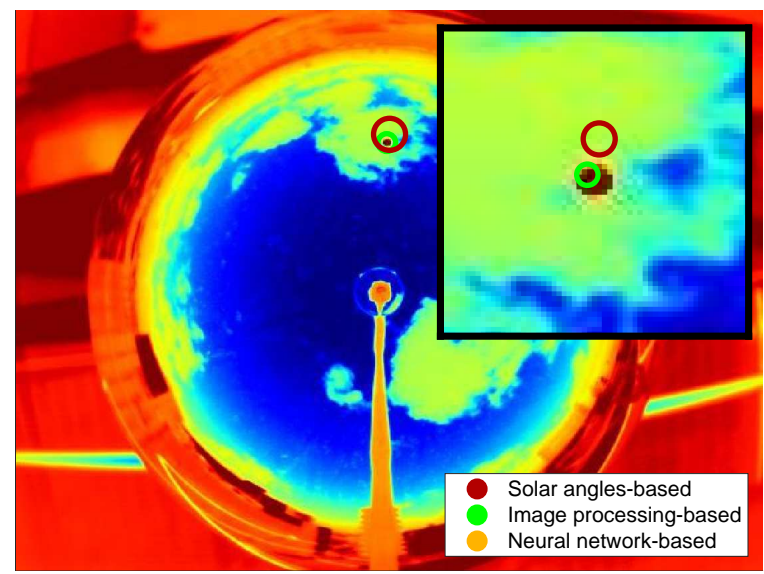

(d) Time: 12:24

Figure 15. Results of the estimation of the Sun trajectory (black line) with image processing: for each image, the orange circle corresponds to the estimated Sun position at that time.

Figure 16 shows one of the rare cases in which identification by means of the image processing fails, providing an unreliable result. Analyzing the zoomed plot, it is possible to see that, while the neural network has been able to avoid this error, due to particular conditions such as the color distortion of the Sun and a spot of color saturation in the reduced elliptical shape, the image processing identified a single 
wrong accepted candidate. Despite those noises in the training dataset, the ANN learning process was able to mitigate their presence without compromising the overall prediction ability. This ability must be further examined by increasing the training dataset to a larger number of days.

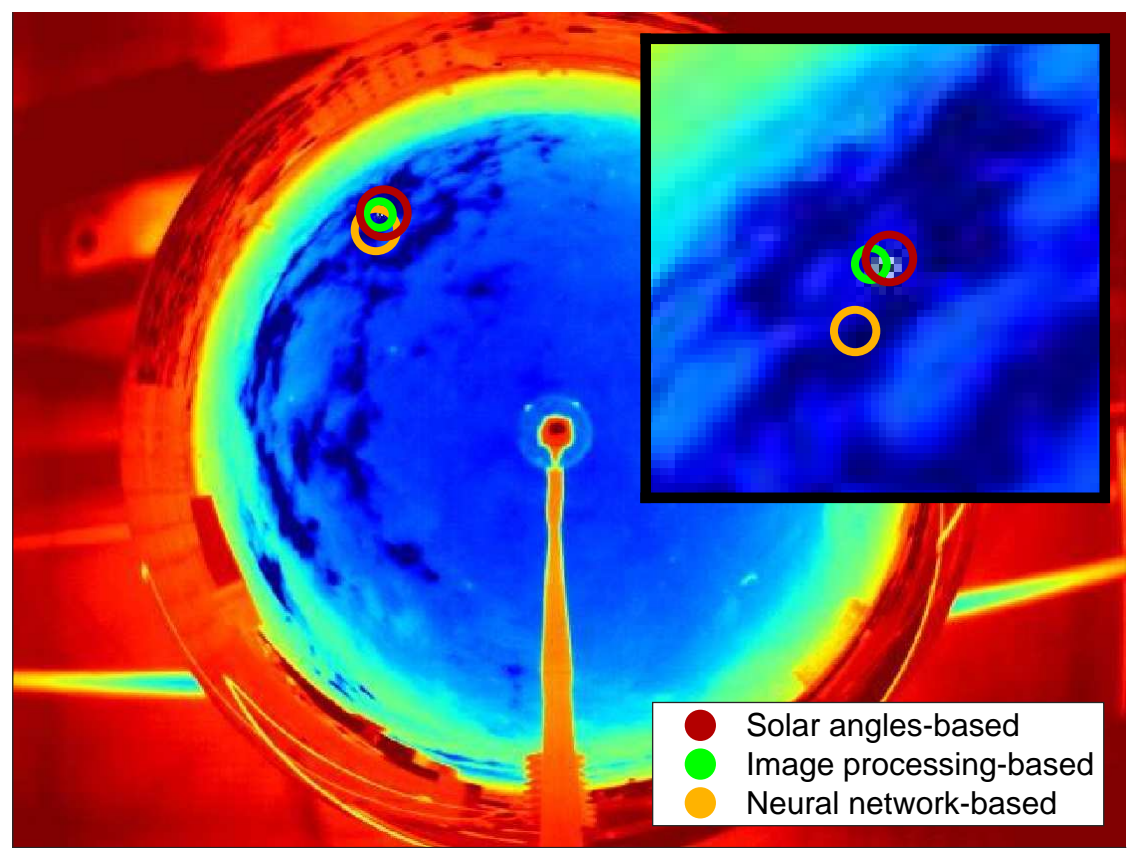

Figure 16. Representation of the structure of the designed Artificial Neural Network (ANN).

\section{Conclusions}

In this paper, three different techniques aiming at identifying the solar position in whole-sky images were proposed, described, and compared. The implemented approaches were specifically set and tailored in order to serve the scope of providing a first step towards the definition of a novel methodology to perform very short-term PV power forecast, namely, nowcasting.

The first method relies on the theoretical solar position in the sky and was described in terms of the solar azimuth and elevation ( $\gamma$ and $\alpha$, respectively). The projection of the theoretical position on the images was affected by approximation due to the partially unknown deformation introduced by the reflection and acquisition system. The second methodology performed identification of the Sun through an image processing algorithm exploiting the chromatic difference existing in the images among the sky, the Sun, and the surroundings. Finally, the third approach used information obtained through the first two methodologies to train an artificial neural network.

These proposed techniques were tested using real images obtained with a camera installed at SolarTech $^{\mathrm{Lab}}$ at Politecnico di Milano, Italy. They were compared in terms of reliability, accuracy, and computational load.

The overall best performances are obtained through the adoption of the ANN-based technique that, thanks to its generalization capability, is able to accurately infer the solar position in a wide spectrum of conditions: in fact, the error committed by this methodology is $0.01 \%$, while the image processing-based method is above $3 \%$. 
Author Contributions: Conceptualization, A.N. (Alessandro Niccolai) and A.N. (Alfredo Nespoli); data curation, A.N. (Alessandro Niccolai) and A.N. (Alfredo Nespoli); formal analysis, A.N. (Alessandro Niccolai) and A.N. (Alfredo Nespoli); methodology, A.N. (Alessandro Niccolai) and A.N. (Alfredo Nespoli); software, A.N. (Alessandro Niccolai) and A.N. (Alfredo Nespoli); supervision, A.N. (Alessandro Niccolai); validation, A.N. (Alfredo Nespoli); visualization, A.N. (Alessandro Niccolai) and A.N. (Alfredo Nespoli); writing-original draft, A.N. (Alessandro Niccolai) and A.N. (Alfredo Nespoli); writing—review and editing, A.N. (Alessandro Niccolai) and A.N. (Alfredo Nespoli). All authors have read and agreed to the published version of the manuscript.

Funding: This research received no external funding.

Conflicts of Interest: The authors declare no conflict of interest.

\section{References}

1. IEA. Renewables 2019-Analysis and Forecast to 2024; IEA: Paris, France, 2019.

2. Marcos, J.; Storkël, O.; Marroyo, L.; Garcia, M.; Lorenzo, E. Storage requirements for PV power ramp-rate control. Sol. Energy 2014, 99, 28-35. [CrossRef]

3. Verbois, H.; Huva, R.; Rusydi, A.; Walsh, W. Solar irradiance forecasting in the tropics using numerical weather prediction and statistical learning. Sol. Energy 2018, 162, 265-277. [CrossRef]

4. Ferlito, S.; Adinolfi, G.; Graditi, G. Comparative analysis of data-driven methods online and offline trained to the forecasting of grid-connected photovoltaic plant production. Appl. Energy 2017, 205, 116-129. [CrossRef]

5. Boyle, G. Renewable Electricity and the Grid: The Challenge of Variability; Routledge: London, UK, 2012; pp. 1-219. [CrossRef]

6. Moretti, L.; Polimeni, S.; Meraldi, L.; Raboni, P.; Leva, S.; Manzolini, G. Assessing the impact of a two-layer predictive dispatch algorithm on design and operation of off-grid hybrid microgrids. Renew. Energy 2019, 143, 1439-1453. [CrossRef]

7. Ogliari, E.; Niccolai, A.; Leva, S.; Zich, R.E. Computational intelligence techniques applied to the day ahead PV output power forecast: PHANN, SNO and mixed. Energies 2018, 11, 1487. [CrossRef]

8. Chen, S.X.; Gooi, H.B.; Wang, M.Q. Sizing of energy storage for microgrids. IEEE Trans. Smart Grid 2012, 3, 142-151. [CrossRef]

9. Gigoni, L.; Betti, A.; Crisostomi, E.; Franco, A.; Tucci, M.; Bizzarri, F.; Mucci, D. Day-Ahead Hourly Forecasting of Power Generation from Photovoltaic Plants. IEEE Trans. Sustain. Energy 2018, 9, 831-842. [CrossRef]

10. Bizzarri, F.; Bongiorno, M.; Brambilla, A.; Gruosso, G.; Gajani, G.S. Model of photovoltaic power plants for performance analysis and production forecast. IEEE Trans. Sustain. Energy 2013, 4, 278-285. [CrossRef]

11. Lee, S.; Jin, H.; Vecchietti, L.F.; Hong, J.; Har, D. Short-Term Predictive Power Management of PV-Powered Nanogrids. IEEE Access 2020, 8, 147839-147857. [CrossRef]

12. Khalili, T.; Jafari, A.; Abapour, M.; Mohammadi-Ivatloo, B. Optimal battery technology selection and incentive-based demand response program utilization for reliability improvement of an insular microgrid. Energy 2019, 169, 92-104. [CrossRef]

13. Mellit, A.; Pavan, A.M.; Ogliari, E.; Leva, S.; Lughi, V. Advanced methods for photovoltaic output power forecasting: A review. Appl. Sci. 2020, 10, 487. [CrossRef]

14. Chen, X.; Du, Y.; Lim, E.; Wen, H.; Jiang, L. Sensor network based PV power nowcasting with spatio-temporal preselection for grid-friendly control. Appl. Energy 2019, 255, 113760. [CrossRef]

15. Khalili, T.; Nojavan, S.; Zare, K. Optimal performance of microgrid in the presence of demand response exchange: A stochastic multi-objective model. Comput. Electr. Eng. 2019, 74, 429-450. [CrossRef]

16. de la Parra, I.; Marcos, J.; García, M.; Marroyo, L. Dealing with the implementation of ramp-rate control strategies-Challenges and solutions to enable PV plants with energy storage systems to operate correctly. Sol. Energy 2018, 169, 242-248. [CrossRef]

17. Oneto, L.; Laureri, F.; Robba, M.; Delfino, F.; Anguita, D. Data-driven photovoltaic power production nowcasting and forecasting for polygeneration microgrids. IEEE Syst. J. 2018, 12, 2842-2853. [CrossRef]

18. Bright, J.M.; Killinger, S.; Lingfors, D.; Engerer, N.A. Improved satellite-derived PV power nowcasting using real-time power data from reference PV systems. Sol. Energy 2018, 168, 118-139. [CrossRef] 
19. Catalina, A.; Alaiz, C.M.; Dorronsoro, J.R. Combining Numerical Weather Predictions and Satellite Data for PV Energy Nowcasting. IEEE Trans. Sustain. Energy 2020, 11, 1930-1937. [CrossRef]

20. Kurtz, B.; Mejia, F.; Kleissl, J. A virtual sky imager testbed for solar energy forecasting. Sol. Energy 2018, 158, 753-759. [CrossRef]

21. Schmidt, T.; Calais, M.; Roy, E.; Burton, A.; Heinemann, D.; Kilper, T.; Carter, C. Short-term solar forecasting based on sky images to enable higher PV generation in remote electricity networks. Renew. Energy Environ. Sustain. 2017, 2, 23. [CrossRef]

22. Almonacid-Olleros, G.; Almonacid, G.; Fernandez-Carrasco, J.I.; Estevez, M.E.; Quero, J.M. A new architecture based on iot and machine learning paradigms in photovoltaic systems to nowcast output energy. Sensors 2020, 20, 4224. [CrossRef]

23. Dutta, S.; Li, Y.; Venkataraman, A.; Costa, L.M.; Jiang, T.; Plana, R.; Tordjman, P.; Choo, F.H.; Foo, C.F.; Puttgen, H.B. Load and Renewable Energy Forecasting for a Microgrid using Persistence Technique. Energy Procedia 2017, 143, 617-622. [CrossRef]

24. Kumler, A.; Xie, Y.; Zhang, Y. A Physics-based Smart Persistence model for Intra-hour forecasting of solar radiation (PSPI) using GHI measurements and a cloud retrieval technique. Sol. Energy 2019, 177, 494-500. [CrossRef]

25. Nespoli, A.; Niccolai, A. Solar Position Identification on Sky Images for Photovoltaic Nowcasting applications. In Proceedings of the IEEE International Conference on Environment and Electrical Engineering and IEEE Industrial and Commercial Power Systems Europe, EEEIC/I and CPS Europe, Madrid, Spain, 9-12 June 2020; pp. 6-10. [CrossRef]

26. SolarTech Lab: Forecasting. Available online: http://www.solartech.polimi.it/activities/forecasting/ (accessed on 3 November 2020).

27. SolarTech Lab: Instrumentation. Available online: http://www.solartech.polimi.it/instrumentation/ (accessed on 3 November 2020).

28. Hughes, G.W. Engineering Astronomy; Tech. Rep. 5323; Sandia National Laboratories: Albuquerque, NM, USA, 1985.

29. Holmgren, W.; Hansen, C.; Mikofski, M. Pvlib Python: a Python Package for Modeling Solar Energy Systems. J. Open Source Softw. 2018, 3, 884. [CrossRef]

Publisher's Note: MDPI stays neutral with regard to jurisdictional claims in published maps and institutional affiliations. 